eISSN 2444-7986

DOI: https://doi.org/10.14201/orl201782.15577

Artículo de revisión

\title{
LA PRESBIFONÍA COMO CAUSA DE INSUFICIENCIA GLÓTICA
}

\section{Presbiphonia as a cause of glotic insufficiency}

\author{
Jhonder Xavier SALAZAR-GUILARTE; Antonio ARROYO-SEBASTIÁN; Juan Ramón GRAS- \\ ALBERT; Francisco SÁNCHEZ-DEL CAMPO
}

Universidad Miguel Hernández de Elche. Programa de Doctorado en Salud Pública, Ciencias Médicas y Quirúrgicas. Alicante. España.

Correspondencia: jhoxasagui@gmail.com

Fecha de recepción: 1 de enero de 2017

Fecha de aceptación: 1 de marzo de 2017

Fecha de publicación: 3 de marzo de 2017

Fecha de publicación del fascículo: 1 de junio de 2017

Conflicto de intereses: Los autores declaran no tener conflictos de intereses

Imágenes: Los autores declaran haber obtenido las imágenes con el permiso de los pacientes

Política de derechos y autoarchivo: se permite el autoarchivo de la versión post-print (SHERPA/RoMEO)

Licencia CC BY-NC-ND. Licencia Creative Commons Atribución-NoComercial-SinDerivar 4.0 Internacional

๔ ) Universidad de Salamanca. Su comercialización está sujeta al permiso del editor

RESUMEN

Introducción y objetivo: El envejecimiento de la voz se ha convertido en una causa frecuente de consulta en la práctica diaria del otorrinolaringólogo, debido entre otras causas al envejecimiento progresivo de la población y sociedades de los países industrializados. Con este trabajo de revisión pretendemos una puesta al día en relación a los cambios clínicos y patológicos de la laringe que conllevan a la presbifonía, abordando los aspectos más relevantes sobre sus causas, manifestaciones clínicas y medidas terapéuticas. Materiales y métodos: trabajo descriptivo, resultado de la búsqueda bibliográfica en la base de datos PubMed con las estrategias de búsqueda [(presbyphonia OR aging voice disorders) AND vocal atrophy]. Se seleccionaron revisiones, ensayos clínicos y artículos en inglés y español de los últimos 10 años. Se excluyeron casos clínicos, artículos de insuficiencia glótica o disfonía por otras causas. Resultados: La revisión sistemática ofreció 34 artículos, de los cuales, 17 cumplieron los criterios de inclusión. Los resultados deben interpretarse con optimismo, a pesar de su variabilidad la presbifonía es un diagnóstico en crecimiento, con mayor auge entre otorrinolaringólogos dedicados a laringología. Conclusiones: Realizamos una revisión sobre los aspectos más relevantes en relación a los hallazgos clínicos, endoscópicos y anatómicos que ocurren en la presbifonía. Presentamos las diferentes formas de tratamiento propuestas hasta ahora para minimizar la insuficiencia glótica y mejorar la competencia vocal en personas envejecidas.

PALABRAS CLAVE presbifonía; desórdenes vocales; envejecimiento; atrofia vocal

SUMMARY

Introduction and objective: The aging of the voice has become a frequent cause of consultation in the otolaryngologist's daily practice, due to the progressive aging of the population and societies of industrialized countries. With this review, we intend to update the clinical and pathological changes of the larynx that lead to presbyphonia, addressing the most relevant aspects about its causes, clinical manifestations and therapeutic measures. Materials and methods: descriptive and narrative literature review, result of the bibliographic search in the database 
PubMed with the descriptors strategies [(presbyphonia OR aging voice disorders) AND vocal atrophy]. Revisions, clinical trials and general articles in both english and spanish were selected for the past 10 years. Clinical cases, articles of glottic insufficiency or dysphonia due to other causes were excluded. Results: The systematic review offered 34 articles, of which, 17 met the inclusion criteria. The results should be interpreted with optimism, despite its variability; the presbyphonia is a growing diagnosis, with a greater rise among otorhinolaryngologists dedicated to laryngology. Conclusions: We perform a review on the most relevant aspects regarding the clinical, endoscopic and anatomical findings that occur in presbyphonia. We present the different forms of treatment proposed so far to minimize glottic insufficiency and improve vocal competence in aged people.

\section{INTRODUCCIÓN}

Las sociedades están envejeciendo significativamente desde finales del siglo pasado, especialmente en países desarrollados como Japón, España o Estados Unidos, donde después del efecto del baby boomer se observa un constante cambio demográfico y aumento de la población envejecida [1]. Buena parte del crecimiento de esta subpoblación puede ser atribuida al avance de la medicina moderna y de la salud pública. Según las estadísticas de la ONU, en el año 2030, los adultos mayores serán casi un tercio de la población mundial, por lo que el manejo de la voz envejecida se convertirá en un problema y motivo de consulta en aumento para el otorrinolaringólogo y los profesionales dedicados al cuidado de la voz [2, 3].

La incidencia de desórdenes funcionales de la voz en personas ancianas es poco conocida, pero ha sido citada del 12 al $35 \%[3,4]$. Hasta ahora no hay datos exactos de la prevalencia de los trastornos vocales en adultos mayores, ya que muchos creen que el problema es parte natural del envejecimiento y pocos buscan cuidado a su problema de voz. La mayoría de los estudios publicados son retrospectivos e identifican la disfonía y no la presbifonía como principal motivo de consulta, o bien se trata de estudios prospectivos, pero con un tamaño de muestra pequeño de pacientes observados y tratados [4].

El proceso de envejecimiento atañe también a las estructuras laríngeas y se viene a manifestar como un conjunto de eventos fisiológicos que terminan por modificar la voz y la laringe, que incluye cambios en los diversos tejidos de las cuerdas vocales, su mucosa, músculos y cartílagos [5]. En ese sentido, hay que hacer una clara diferenciación entre presbifonía y presbidisfonía, palabra etimológicamente derivada del griego (presbites: anciano y dys-phonia alteración en la articulación vocal) que no es más que la disfonía que ocurre en adultos de la tercera edad, y en donde las lesiones benignas tales como los pólipos siguen siendo la causa más común, seguida de lesiones malignas, parálisis de cuerdas vocales, disfonía funcional, desórdenes neurológicos, condiciones inflamatorias debido a reflujo faringolaríngeo, entre otras. Contrario a esto, la situación clínica en la que adultos mayores se presentan al otorrinolaringólogo con una complicada aglomeración de síntomas, entre los que destacan tiempos de fonación acortados, voz de tipo aérea con cierta humedad, debilitamiento en la proyección, y cambios en el timbre de voz, es conocido como presbifonía, y es un fenómeno biológico cuyo diagnóstico debe ser hecho por exclusión y apoyado por hallazgos físicos luego de haber descartado otras comorbilidades, en especial aquellas relacionadas con el pulmón, que afectan directamente sobre la presión glótica y el volumen vocal [1, 2].

En este grupo etario hay una clara correlación entre la percepción de la calidad de vida individual (operatividad física, vitalidad, salud general, salud mental, dolor corporal y papel físico en la vida) con la percepción de la calidad de la voz, que trae como consecuencia que esta población vea su comunicación afectada negativamente y evite situaciones sociales que incluyan un uso excesivo de su voz. Es tal, que un amplio número de personas mayores que cantan en coros pueden ver limitados sus rendimientos, abandonando dicha actividad y privándose de momentos de relajación y ocio; o que profesionales de la voz, como profesores, puedan terminar tempranamente sus actividades por dichas limitaciones [2]. Además, con frecuencia las personas mayores están en contacto con personas contemporáneas que presentan déficits auditivos, por lo que deben hacer un esfuerzo mayor para ser oídos, poniendo en evidencia estos trastornos de la voz [6]. 
La percepción acústica alterada de su voz, combinada con el esfuerzo y dificultad para tratar de emitirla correctamente aumentan la ansiedad y la frustración, lo que termina adversamente afectando su calidad de vida [4, 6].

Con este trabajo de revisión pretendemos una puesta al día en relación a los cambios clínicos y patológicos de la laringe que conllevan a la presbifonía, abordando los aspectos más relevantes sobre sus causas, manifestaciones clínicas y medidas terapéuticas.

\section{MATERIAL Y MÉTODO}

Se trata de una revisión narrativa y descriptiva, resultado de la búsqueda bibliográfica en la base de datos PubMed con las estrategias de búsqueda [(presbyphonia OR aging voice disorders) AND vocal atrophy]. Se seleccionaron revisiones, ensayos clínicos y artículos en inglés y español de los últimos 10 años. Se excluyeron casos clínicos, artículos de insuficiencia glótica o disfonía por otras causas. La revisión ofreció 34 artículos, de los cuales, 17 cumplieron los criterios de inclusión.

\section{CAUSAS}

La producción de la voz óptima conocida como eufonía depende de tres sistemas (respiratorio, vibratorio y resonador) que se afectan claramente con el devenir de la edad, sin embargo, debe tenerse claro que el envejecimiento por sí solo no es la causa más frecuente de disfonía en la población envejecida $[1,2]$.

Los problemas de voz en los adultos mayores pueden resultar del proceso de envejecimiento estructural y neurogénico normal de la laringe que afecte el mecanismo de producción de la voz o ser el resultado de procesos patológicos relacionados con el mal uso y la sobrecarga vocal, y/o con la condición de salud física y psicosocial de la persona. También es posible encontrar soslayados ambos procesos, es decir, que interactúen procesos patológicos sobre la base de laringes envejecidas, observable, por ejemplo, en pacientes que intentan compensar el deterioro vocal propio de la edad adoptando unos patrones de voz hiperfuncionantes, lo que daría lugar de forma crónica a la generación de nódulos. En cualquier caso, dada la dificultad de conocer el verdadero origen, es probable que los trastornos de voz en adultos mayores sea una entidad multifactorial y que un grado más bajo en el uso de la voz se co- rrelacione con trastornos de descondicionamiento muscular tales como curvado y atrofia de la cuerda vocal, propios de la presbifonía [7].

Actualmente, aunque la base científica basada en la evidencia no está totalmente dilucidada, se ha llegado a un consenso sobre los diferentes cambios histológicos y morfológicos en el tejido conectivo de las cuerdas vocales, cuya cascada de acontecimientos se resumiría de la siguiente forma: a) reducción progresiva de la activación de fibroblastos en las cuerdas vocales con la producción de menos ácido hialurónico y de un colágeno más denso -aumentan las fibras de colágeno tipo I- al producido en las cuerdas vocales más jóvenes; b) el epitelio que las recubre disminuye su densidad celular por lo que la matriz extracelular pierde su disposición arquitectónica primaria, y c) se sufre un adelgazamiento y arqueamiento de las cuerdas vocales al mismo tiempo que se hacen más duras y menos móviles. Se cree que el mecanismo de estos cambios viene dado por la acción de las fuerzas mecánicas únicas que sufren estos tejidos durante la oscilación/vibración con el paso del tiempo, resultando en cambios estructurales permanentes [5].

Además de los cambios de la lámina propia y del epitelio, la laringe envejecida, conocida como presbilaringe, presenta calcificación de los cartílagos hialinos, atrofia de sus músculos en general, incluyendo una reducción de las fibras tipo II del músculo tiroaritenoideo que se infiltra por tejido graso, la atrofia de sus glándulas mucosas, degeneración de la articulación cricoaritenoidea y la transmisión nerviosa errática y fallida que conlleva a un desorden en la descarga neuromuscular $[1,2,5]$. No hay que olvidar otros factores extralaríngeos que también contribuyen a estos cambios, como es el aumento de la longitud de la cavidad oral por el adelgazamiento de sus paredes, y por tanto del volumen total del tracto vocal, así como la frecuente pérdida de piezas dentarias que interfiere en la articulación de las palabras y dificulta la inteligibilidad al hablar.

\section{HALLAZGOS CLÍNICOS}

Los cambios en la voz relacionados con la edad son tan marcados que pueden fácilmente revelar la edad de la persona con la que se está hablando por teléfono, sólo al escuchar su voz. El grado de deterioro vocal está relacionado con susceptibilidades individuales tales 
como su salud física y psicológica, hábitos de vida y factores hereditarios y raciales $[2,8]$. En general, los pacientes se quejan de no poder aclarar su garganta, aumento de secreciones en la vía aérea, de la fatiga vocal, poca proyección vocal, fonación de duración acortada, cambios en el tono e incluso aspereza vocal, todo lo cual, aumenta la dificultad de ser oídos cuando hay ruido de fondo, correlacionándose con niveles de espectro vocal aumentados que causan un deterioro progresivo de su calidad

de voz a lo largo del día [1, 8].

La endoscopia convencional es una buena prueba diagnóstica para una evaluación inicial de la insuficiencia glótica en estos pacientes, en donde se aprecia una concavidad de los bordes libres de sus cuerdas vocales que deja un espacio o brecha glótica en la fonación, rigidez de la cuerda vocal y prominencia aumentada de la apófisis vocal de los cartílagos aritenoides [9]. El nivel de concavidad de la cuerda vocal no se relaciona con el grado de gap glótico al momento de la fonación, lo que indica que un elemento de compensación puede estar presente en algunos individuos [10].

Asimismo, la osificación de los cartílagos laríngeos con el paso del tiempo y los cambios degenerativos de los tendones que se fijan a esos cartílagos puede afectar las propiedades viscoelásticas de las estructuras vibratorias de la laringe y producir los cambios aerodinámicos característicos de la voz. El espacio ventricular se encuentra dilatado, y las bandas ventriculares pueden hipertrofiarse en un intento por compensar el pobre cierre glótico [11]. Las glándulas secretoras de moco de la laringe cambian pudiendo resultar en lubricación de peor calidad y menor cantidad que puede afectar el correcto movimiento mucociliar y la remoción efectiva de irritantes de la superficie laríngea. Ocasionalmente, si la atrofia de las cuerdas vocales es severa, puede el paciente referir disfagia e incluso aspiración $[10,11]$.

Los cambios aerodinámicos documentados están estrechamente ligados al estado pulmonar (presión espiratoria pulmonar y capacidad vital) e incluyen la reducción del tiempo máximo de fonación, aumento de la tasa de flujo aéreo a través de la glotis, y disminución del número de sílabas por espiración efectiva [8]; mientras que los cambios electromiográficos incluyen el aumento en la duración y la amplitud de la unidad motora y una disminución en los potenciales de acción de descarga de los músculos laríngeos intrínsecos puesta en evidencia en la inestabilidad y el tremor vocal propia de estos pacientes [8, 9].

También se ha demostrado que la progresión de los cambios que van apareciendo en el envejecimiento vocal no es uniforme desde la edad que comienza, pasando muchas veces desapercibidos mientras la relación con sus allegados sea posible, impactando a la larga negativamente en la calidad de vida en los afectados, y siendo peor en personas con salud deteriorada por otros motivos [6]

\section{TRATAMIENTO}

Como se comentó previamente la presbifonía es un diagnóstico de exclusión y debe ser abordado considerando múltiples factores. La terapia de la voz, representa la primera línea de ataque y juega un papel crítico ya que reduce los comportamientos compensatorios de mal adaptación del aparato fonatorio y aumenta la consistencia de la calidad de la voz. Contempla ejercicios de fortalecimiento para el control respiratorio y fonatorio mediante la educación vocal y postural aumentando la coordinación neuromuscular de todo el conjunto. Esto lleva a la mejora en la comunicación oral mediante la disminución del esfuerzo al hablar y el ajuste de la calidad vocal en función de las necesidades de la edad [12].

Los ejercicios vocales han sido propuestos para favorecer el cierre glótico, aumentar la presión subglótica y la intensidad de la voz, estabilizar la calidad vocal y la frecuencia fundamental, además de proveer una mejoría global del habla. La rehabilitación en la voz puede mejorar la calidad de la voz, tiempo máximo fonatorio, coordinación vocal y respiratoria, tensión, articulación, velocidad, intensidad, resonancia, tono y ataque vocal, dependiendo del objetivo de la misma. Estos ejercicios deben empezar por mejorar el sistema respiratorio, controlar la resonancia y lograr una hiperfunción supraglótica para hacer contactar las cuerdas vocales. Los protocolos deben abarcar ejercicios fonatorios de alta intensidad para sobrecargar el sistema respiratorio y laríngeo y mejorar la intensidad vocal y reducir el esfuerzo vocal. Siempre se debe recomendar la terapia de voz inicialmente cuando se haya determinado que la presbilaringe es la causa del cambio de voz $[12,13]$.

Los fallos en la terapia de voz se deben principalmente a que se requiere de múltiples visitas 
al rehabilitador que muchas veces no se pueden cumplir asociado la mayoría de veces a pacientes con atrofia de cuerdas vocales severa o que cursan con enfermedad sistémica, especialmente pulmonar [13].

Cuando la terapia de la voz falla la cirugía puede mejorar el cierre glótico, mediante el aumento del espacio paraglótico que medializa las cuerdas vocales, procedimiento frecuente en el tratamiento de las parálisis recurrentes de las cuerdas vocales. Sin embargo, un paso intermedio lo representa de forma temporal la laringoplastia de inyección o glotoplastia, cuya finalidad es aumentar el volumen de la glotis y mejorar su cierre. Múltiples sustancias han sido inyectadas profundas al ligamento vocal, e incluyen Teflon $®$, silicona, grasa, Gelfoam $®$, colágeno, ácido hialurónico, carboximetilcelulosa, e hidroxipatita de calcio, bien en la consulta o bajo laringoscopia directa en el quirófano [14]. Debido a que la presbilaringe es básicamente una patología bilateral, se deben tratar ambas cuerdas vocales, sin embargo, una de las principales limitaciones en el uso de estas sustancias es la poca permanencia del efecto deseado lo que requiere de subsecuentes procedimientos. Por otra parte, el inconveniente de hacerlo en la consulta es que el procedimiento requiere de una curva de aprendizaje para el abordaje, bien realizando la inyección mediante nasofibroscopio de doble canal, vía transoral o vía transcervical, todas tienen la ventaja de comprobar la voz in situ, y de evitar los riesgos quirúrgicos de una población ya de por sí susceptiblemente frágil para procedimientos en el quirófano con anestesia general. La inyección del ácido hialurónico en las cuerdas vocales mejora la calidad de la voz en pacientes con insuficiencia glótica $[14,15,16]$.

La cirugía, conocida como tiroplastia tipo 1 o de medialización, está destinada a casos donde hay una brecha glótica amplia y un adelgazamiento severo de la cuerda vocal. Esta técnica permanente requiere abordaje cervical externo e introducción de materiales de diferente composición, forma y tamaño a través de una ventana en el cartílago tiroides.

Los pacientes pueden beneficiarse de una terapia de voz postquirúrgica con la intención de maximizar la función laríngea, manteniendo una adecuada calidad vocal y guiando a los pacientes a dejar hábitos que son dañinos para la voz y adoptando estrategias de prevención. Los pacientes con disfunción pulmonar y gap glótico también se pueden beneficiar de terapia de la voz y ejercicios respiratorios, así como broncodilatadores.

Con el avance del conocimiento de la composición de las cuerdas vocales, se vislumbra un futuro prometedor para tratar la insuficiencia glótica característica de la presbifonía mediante la inyección de fibroblasto cultivado, ya que estas células son componentes esenciales de las cuerdas vocales y responsables de la reparación fisiológica de su matriz extracelular. Se necesitan más estudios e investigación para probar los posibles beneficios de estas nuevas técnicas terapéuticas [17].

\section{CONCLUSIONES}

La presbifonía se asocia a cambios clínicos, endoscópicos y anatómicos que pueden abordarse con distintas terapias.

Aún quedan muchos aspectos por conocer e investigar sobre los cambios que se producen en la laringe y su variabilidad relacionados con el envejecimiento que afecta a la calidad de la voz.

\section{AGRADECIMIENTOS}

A Encarni Aguilar, de la biblioteca del Hospital General Universitario de Alicante, por su valiosa y desinteresada ayuda en la búsqueda de material bibliográfico.

\section{BIBLIOGRAFÍA}

1. Kendall K. Presbyphonia: a review. Curr Opin Otolaryngol Head Neck Surg. 2007;15(3):137-40.

2. Martins RH, Gonçalvez TM, Pessin AB, Branco A. Aging voice: presbyphonia. Aging Clin Exp Res. 2013;26(1):1-5.

3. Roy N, Stemple J, Merrill RM, Thomas L. Epidemiology of Voice Disorders in the Elderly: Preliminary Findings. Laryngoscope. 2007;117(4):628-33.

4. Golub JS, Chen PH, Otto KJ, Hapner E, Johns MM 3rd. Prevalence of Perceived Dysphonia in a Geriatric Population. J Am Geriatr Soc. 2006;54(11):1736-9.

5. Martins $\mathrm{RH}$, Benito Pessin $A B$, Nassib DJ, Branco A, Rodrigues SA, Matheus SM. Aging voice and the laryngeal muscle atrophy. Laryngoscope. 2015;125(11):251821. 
6. Zraick R, Risner B. Assessment of quality of life in persons with voice disorders. Curr Opin Otolaryngol Head Neck Surg. 2008;16(3):188-193.

7. Bastian R, Thomas J. Do Talkativeness and Vocal Loudness Correlate With Laryngeal Pathology? A Study of the Vocal Overdoer/Underdoer Continuum. J Voice. 2016;30(5):557-62.

8. Vaca M, Mora E, Cobeta I. The Aging Voice: Influence of Respiratory and Laryngeal Changes. Otolaryngol Head Neck Surg. 2015;153(3):409-13.

9. Vaca M, Cobeta I, Mora E, Reyes P. Clinical Assessment of Glottal Insufficiency in Age-related Dysphonia. J Voice. 2017;31(1):128.e1-128.e5.

10. Johns M, Arviso L, Ramadan F. Challenges and Opportunities in the Management of the Aging Voice. Otolaryngol Head Neck Surg. 2011;145(1):1-6.

11. Takano S, Kimura M, Nito T, Imagawa $\mathrm{H}$, Sakakibara K, Tayama N.Clinical analysis of presbylarynx-Vocal fold atrophy in elderly individuals. Auris Nasus Larynx. 2010;37(4):461-4.
12. Ziegler A, Verdolini Abbott $K$, Johns $M$, Klein A, Hapner ER. Preliminary data on two voice therapy interventions in the treatment of presbyphonia. Laryngoscope. 2014;124(8):1869-76.

13. Mau T, Jacobson B, Garrett C. Factors associated with voice therapy outcomes in the treatment of presbyphonia. Laryngoscope. 2010;120(6):1181-7.

14. Bradley J, Hapner E, Johns M. What is the optimal treatment for presbyphonia?. Laryngoscope. 2014;124(11):2439-40.

15. Benninger $M$, Hanick $A$, Nowacki $A$. Augmentation Autologous Adipose Injections in the Larynx. Ann Otol Rhinol Laryngol. 2015;125(1):25-30.

16. Miaśkiewicz $B$, Szkiełkowska A, Piłka A, et al. Assessment of acoustic characteristics of voice in patients after injection laryngoplasty with hyaluronan. Otolaryngol Pol. 2016;70(1):15-23.

17. Hirano S, Tateya I, Kishimoto $\mathrm{Y}$, Kanemaru S, Ito J. Clinical trial of regeneration of aged vocal folds with growth factor therapy. Laryngoscope. 2012;122(2):327-31. 